\title{
Turbid Challenge in Diabetic Ketoacidosis
}

\author{
Nandita M Jindal ${ }^{1}$, Maria Thomas ${ }^{2}$, Ashish K Joy ${ }^{3}$
}

\begin{abstract}
Background: Interference due to lipemia poses major challenges in laboratory analysis. Turbidity in the sample is due to the accumulation of triglyceride (TG)-rich lipoprotein particulates. The most common cause of hypertriglyceridemia is inadequate fasting whereas secondary causes include alcoholism, chronic renal failure, hypothyroidism, and diabetes mellitus.

Aim and objective: To analyze a highly lipemic sample received in the laboratory, to aid in diagnosis.

Case description: A highly lipemic plasma drawn from a previously well 32-year-old woman presenting to the emergency department with fever, vomiting, breathlessness, and epigastric pain was received in the lab. Increased serum cholesterol (1,067 mg/dL) with highly raised TG levels $(16,117 \mathrm{mg} / \mathrm{dL})$ was seen. Insulin was found to be low $(4.2 \mu \mathrm{l} / \mathrm{mL})$ with raised blood glucose $(379 \mathrm{mg} / \mathrm{dL})$ and serum was found positive for acetone. Initial serum lipoprotein electrophoresis showed prominent bands of very-low-density lipoprotein (VLDL) and chylomicrons, which after the start of insulin treatment were found to be only of VLDL. The patient was managed as a case of diabetic ketoacidosis subsequently resulting in a fall in the levels of TG $(1,022 \mathrm{mg} / \mathrm{dL})$ and glucose $(176 \mathrm{mg} / \mathrm{dL})$ and negative serum acetone.

Conclusion: We report the approach taken by the laboratory toward a highly turbid serum sample to achieve reliable laboratory results from a patient eventually diagnosed with diabetic ketoacidosis and acute pancreatitis.

Clinical significance: Diagnosis of a patient in current times is mostly dependent on accurate laboratory results which are affected by preanalytical variables like lipemia. Appropriate sample handling in such cases as well as using techniques at our disposal like lipid electrophoresis can aid in diagnosis and ruling out differential causes.
\end{abstract}

Keywords: Diabetic ketoacidosis, Electrophoresis, Hypertriglyceridemia, Lipemia, Lipoproteins.

Indian Journal of Medical Biochemistry (2021): 10.5005/jp-journals-10054-0185

\section{BACKGROUND}

Interference due to lipemia poses major challenges in laboratory analysis. It is a frequent and yet unresolved problem in clinical chemistry. ${ }^{1}$ It affects most spectrophotometric, colorimetric, and indirect potentiometric tests. Methods using lower wavelengths are most affected such as urea, creatinine, gamma-glutamyl transferase (GGT), aspartate transaminase (AST), alanine transaminase (ALT), and amylase. ${ }^{2}$ In cases where hypertriglyceridemia is not related to fasting and has a direct association with the underlying disease, appropriate sample processing and downstream analysis is essential for reliable reporting. Various techniques described for the resolution of lipemic interferences are ultracentrifugation, sample dilutions, and extractions using polar solvents. ${ }^{2}$ Without these corrective methods, it is not possible to release useful and reliable lab results which will help in the diagnosis and monitoring of patients.

\section{Case Description}

The laboratory received a highly lipemic plasma (Fig. 1) drawn at the time of admission from a 32-year-old woman presenting with fever, non-projectile vomiting, breathlessness, and constipation for 2 days. She had epigastric pain which was moderate in intensity and non-radiating. Initial assessment showed a regular pulse rate of 92/ minute, respiratory rate of $20 /$ minute, and blood pressure of $110 / 70$ $\mathrm{mm} \mathrm{Hg}$. Her medical history revealed cholelithiasis and untreated gestational diabetes. There was no history of polydipsia or polyuria and no similar episodes previously. Physical examination showed no evidence of xanthoma, xanthelasma, or eruptive xanthoma. USG abdomen showed calculous cholecystitis. Ophthalmic examination showed signs of lipemia retinalis. She was not on any medication.
${ }^{1}$ Department of Biochemistry, Dayanand Medical College and Hospital, Ludhiana, Punjab, India

${ }^{2}$ Department of Biochemistry, Christian Medical College and Hospital, Ludhiana, Punjab, India

${ }^{3}$ Department of Biochemistry, Travancore Medical College, Kollam, Kerala, India

Corresponding Author: Nandita M Jindal, Department of Biochemistry, Dayanand Medical College and Hospital, Ludhiana, Punjab, India, Phone: +91 8427664636, e-mail: dr.nanditamaini@gmail.com

How to cite this article: Jindal NM, Thomas M, Joy AK. Turbid Challenge in Diabetic Ketoacidosis. Indian J Med Biochem 2021;25(2):88-90.

Source of support: Nil

Conflict of interest: None

There was no family history of diabetes, pancreatitis, dyslipidemias, or any chronic diseases.

\section{Materials and Methods}

The lactescent plasma was centrifuged at 3,000 rpm for 15 minutes. The serum obtained was curdy. Most of the lab tests suffered direct interference due to this. A high-speed centrifuge at 15,000 rpm for 30 minutes led to a triglyceride (TG)-rich creamy layer which was carefully removed leaving clear serum below. Further tests were performed on this clear sample.

Several biochemical tests were done keeping various causes of hypertriglyceridemia in mind. All investigations were performed on automated analyzer Cobas 6000 by Roche diagnostics. Before highspeed centrifugation, all parameters showed an error flag against 


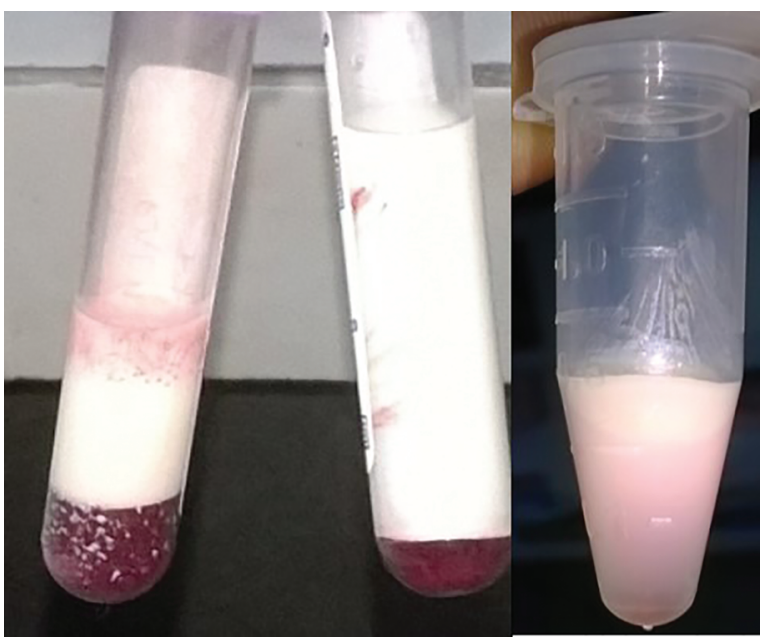

Fig. 1: Lipemic plasma

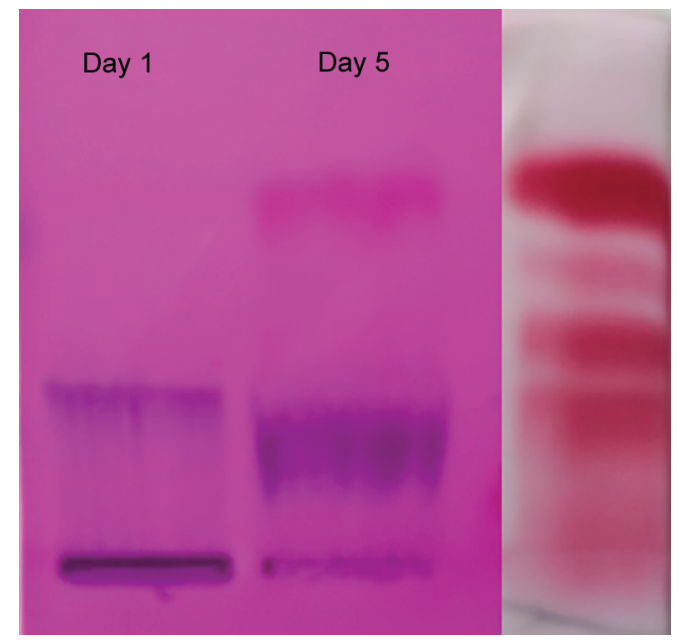

Fig. 2: Day 1 and day 5 lipoprotein electrophoresis with serum protein electrophoresis for comparison

Table 1: Investigations

\begin{tabular}{|c|c|c|c|c|c|c|c|c|}
\hline Test & Day 1 & Day 2 & Day 3 & Day 4 & Day 5 & Day 6 & Day 7 & Day 8 \\
\hline TG & 16,118 & 11,429 & 3,070 & 1,130 & 1,164 & 600 & 1,065 & 1,022 \\
\hline $\mathrm{HDL}$ & 60 & 54 & 60 & 35 & 37 & 31 & 24 & 22 \\
\hline LDL & 52 & 124 & 170 & 206 & 319 & 408 & 374 & 320 \\
\hline T. cholesterol & 1,067 & 369 & 715 & 481 & 605 & 552 & 556 & 515 \\
\hline Glucose & 380 & 308 & 269 & 245 & 210 & 201 & 198 & 176 \\
\hline Acetone & Positive & Positive & Positive & Negative & & & & \\
\hline $\mathrm{NA}^{+}$ & - & 136 & 136 & 149 & 164 & 157 & 149 & 145 \\
\hline $\mathrm{K}^{+}$ & - & 2.42 & 2.5 & 2.8 & 4.3 & 3.3 & 3.4 & 3.7 \\
\hline $\mathrm{Cl}^{-}$ & - & 86 & 97 & 115 & 137 & 130 & 113 & 118 \\
\hline Amylase & 36.9 & - & - & - & - & - & - & - \\
\hline Lipase & 87.7 & - & - & - & - & - & - & - \\
\hline Urea & - & 27 & 53 & 72 & 102 & 91 & 85 & 73 \\
\hline Creatinine & - & 1.5 & 2.8 & 2.58 & 2.9 & 2.1 & 2 & 1.2 \\
\hline
\end{tabular}

results. After centrifugation, all parameters were repeated. Lipid profile was done daily in serial dilutions as well as after high-speed centrifugation. HbA1c was estimated on the Biorad D10 analyzer.

Lipoprotein electrophoresis was performed, using Schiff's reagent to track lipoprotein band, and a simultaneously run serum protein electrophoresis tracked with Ponceau $S$ stain was used for comparison (Fig. 2).

\section{Discussion}

In lipemia, the sample develops turbidity due to the accumulation of TG-rich lipoprotein particulates. TG-rich lipoproteins such as chylomicrons and very-low-density lipoprotein (VLDL) have the greatest potential in causing turbidity in the sample. The most common cause of hypertriglyceridemia is inadequate fasting. Secondary causes include alcoholism, chronic renal failure (CRF), diabetes mellitus (DM), hypothyroidism, and pancreatitis., ${ }^{1,2}$

In our patient, human chorionic gonadotropin ( $\beta$-HCG) was found to be normal $(<0.1 \mathrm{mlU} / \mathrm{mL})$, ruling out ectopic pregnancy. Liver function tests (LFT) including total and direct bilirubin, total protein, albumin, alkaline phosphatase (ALP), GGT, ALT, and AST were within normal limits. Thyroid-stimulating hormone (TSH) $(1.1 \mu \mathrm{IU} / \mathrm{mL})$ was found to be insignificant. Cortisol was within the normal range $(7.2 \mu \mathrm{g} / \mathrm{dL})$ and insulin was found to be low $(4.2 \mu \mathrm{lU} /$ $\mathrm{mL}$, reference range: $2.6-24.9 \mu \mathrm{lU} / \mathrm{mL}$ ). $\mathrm{HbA1c}$ was estimated on the Biorad D10 analyzer and found to be $14.9 \%$. Amylase $(36.9 \mathrm{IU} / \mathrm{mL}$, reference range: $28-100 \mathrm{IU} / \mathrm{mL}$ ) was within range and lipase $(87.7$ $\mathrm{IU} / \mathrm{mL}$, reference range $13-60 \mathrm{IU} / \mathrm{mL}$ ) was slightly raised. Initial and subsequent laboratory findings are shown in Table 1. Lipid profiles of siblings of the patient were found to be normal, making familial combined hypertriglyceridemia unlikely.

Lipoprotein electrophoresis revealed VLDL and chylomicrons as the major lipid fractions on day 1 sample with undetectable high-density lipoprotein (HDL) and low-density lipoprotein (LDL) bands. However, the day 5 sample after initiation of insulin therapy showed the disappearance of VLDL and chylomicron bands and the appearance of broad beta and beta fractions corresponding to intermediate-density lipoprotein (IDL) and LDL and a fast-moving HDL band.

Insulin is essential for the activation of the endotheliumassociated lipoprotein lipase (LPL). Lipoprotein lipase hydrolyzes the TG contained in chylomicrons and VLDL. Hence, absolute 
insulin deficiency results in delayed clearing of these particles. Insulin deficiency is usually accompanied by an increase in hormones such as glucagon and epinephrine. These counterregulatory hormones activate the hormone-sensitive lipases in the adipose tissue and bring about the breakdown of TG's present. The resulting free fatty acids flood the circulation from where part of it is taken to the liver and is re-esterified and packaged into VLDL molecules which then flood the circulation. This explains the VLDL and chylomicron band seen in lipid electrophoresis in the first-day sample.

Several studies implicate the role of hypertriglyceridemia resulting in acute pancreatitis (AP); the risk increasing significantly when TG levels rise above $1,000 \mathrm{mg} / \mathrm{dL}$. ${ }^{3,4}$ Free fatty acids are toxic to the pancreatic tissue where they evoke an inflammatory response and activation of enzymes which could explain the symptoms of acute abdominal pain. Amylase and lipase were not significantly raised. However, AP in the setting of DKA with normal lipase levels has also been previously described ${ }^{5}$ as in our case. The relevance of amylase levels is also less specific in such cases due to disturbance in calorimetric measurement. ${ }^{6}$

The patient was managed as a case of diabetic ketoacidosis subsequently resulting in a fall in the levels of TG $(1,022 \mathrm{mg} / \mathrm{dL})$ and glucose (176 mg/dL) and negative serum acetone.

\section{Conclusion}

We report the approach taken by the laboratory toward a highly lipemic serum sample from a patient eventually diagnosed with diabetic ketoacidosis and AP to identify the type of lipoproteinemia and how we achieved reliable laboratory results which were otherwise not achievable on the turbid serum.

\section{Clinical Significance}

Diagnosis of a patient in current times is mostly dependent on accurate laboratory results which are affected by preanalytical variables like lipemia. Appropriate sample handling in such cases as well as using techniques at our disposal like lipid electrophoresis can aid in diagnosis and ruling out differential causes.

With acute presentation and with no previous episodes reported in this patient and the presence of normal reports in siblings, hypertriglyceridemia, in this case, was precipitated by DKA and not familial hypertriglyceridemia.

\section{References}

1. Calmarza $P$, Cordero J. Lipemia interferences in routine clinical biochemical tests. Biochem Med 2011;21(2):160-166. DOI: 10.11613/ bm.2011.025.

2. Nokilac N. Lipemia: causes, interference mechanisms, detection and management. Biochem Med 2014;24(1):57-67. DOI: 10.11613/ BM.2014.008.

3. Singla AA, Ting F, Singla A. Acute pancreatitis secondary to diabetic ketoacidosis induced hypertriglyceridemia in a young adult with undiagnosed type 2 diabetes. JOP 2015;16(2):201-204. DOI: 10.6092/1590-8577/2961.

4. Kota S, Jammula S, Kota S, et al. Acute pancreatitis in association with diabetic ketoacidosis in a newly diagnosed type 1 diabetes mellitus patient; case based review. IJCCI 2012;4:54-60.

5. Argueta EE, Nugent KM. Acute pancreatitis in a patient with diabetic ketoacidosis and normal lipase levels. ICU Director 2013;4(4):166-169. DOI: 10.1177/1944451613488419.

6. Yadav D, Nair S, Norkus E, et al. Nonspecific hyperamylasemia and hyperlipasemia in diabetic ketoacidosis: incidence and correlation with biochemical abnormalities. Am J Gastroenterol 2000;95(11):3123-3128. DOI: 10.1111/j.1572-0241.2000.03279.x. 